\title{
Propriale und nichtpropriale Sprachareale: Einheit in der Vielfältigkeit
}

\author{
Stanislava Kloferová \\ Institut für tschechische Sprache der Akademie der Wissenschaften \\ derTschechischen Republik, CS-Brno, kloferova@iach.cz
}

SCN III/1 [2010], 52-60

Lastno- in občnoimenski areali so odraz jezikovnega delovanja in to je potrebno upoštevati, čeprav med njimi obstajajo razlike (enkratno proti splošno). Prispevek prikazuje, kako lahko odnos med središčem in obrobjem občnoimenskega areala pomaga pri lingvistični analizi negotovih, izoliranih nenaselbinskih lastnih imen (anojkonimov) gre za pomoč pri ugotavljanju njihovega izvora.

Proprial and non-proprial areals are manifestations of how one language functions. Despite differences between them (unique vs. general), this fact can be mutually made use of. The contribution demonstrates how the relation between the nucleus and the periphery of a non-proprial areal can help in linguistic analysis of uncertain, isolated proper names (anoikonyms) and how it can contribute towards finding their origin.

Ključne besede: dialektologija, onomastika, jezikovni areal, ljudska etimologija

Key words: dialektology, onomastics, linguistic areal, folk etymology

\section{Das Areal: Abgrenzung des Termins}

Das Areal als Grundterminus der Areallinguistik wird als eine Begrenzung einer bestimmten Spracherscheinung / bestimmter Spracherscheinungen in deren territorialen Gruppierung / Zuordnung begriffen. Die Existenz einer Arealgrenze und ihr Verlauf erweisen sich dabei als Ergebnis der Wirkung vieler Faktoren, die sich an der Entwicklung der menschlichen Gesellschaft beteiligten: das Sprachareal ist demzufolge eine komplexe linguistische und historische Kategorie. 


\section{Das Areal auf der Sprachkarte}

Die Existenz der Sprachareale wird, wie bekannt, durch eine Sprachkarte gut bezeugt und illustriert: Hier wird / werden eine Spracherscheinung / mehrere Spracherscheinungen in ihrer geographischen Distribution aufgezeichnet. Stehen mehrere Sprachkarten (im Idealfall ein Sprachatlas) zur Verfügung, kann man den Durchlauf mehrerer Grenzen auf einer synthetisierenden Karte vergleichen und dabei feststellen, ob und wie weit sie sich in einem Bündel überdecken bzw. aufeinander schichten. Je umfangreicher der Komplex von Sprachkarten ist, desto größer ist die Chance, die Existenz der Isoglossenbündel (bzw. Staffeln) zu entdecken. Mit der steigenden Anzahl der Isoglossen im Bündel kann man auf die Existenz einer früheren Grenze in der Kommunikation zwischen zwei angrenzenden Sprachgemeinschaften schließen.

\section{Isoglossenbündel als Repräsentant einer Sprach-/dialektalen Grenze}

Die Möglichkeit, so abgegrenzte Areale bzw. Scheiden zu zeigen, hat eine Reihe von dialektalen Atlaswerken ausgenutzt. Erwähnt seien Werke wie der Atlas der slowakischen Sprache mit den Isoglossenbündeln auf den sog. ,synthetischen Karten“ im 1. (Štolc, Buffa, Habovštiak: 1968) und 4. (Habovštiak: 1984) Band, weiters der Sorbische Sprachatlas (Faßke, Jentsch, Michalk: 1965-1996) mit den Karten der Staffeln und Bündel in Bänden 1-14 (im 12. und 13. Band treten darüberhinaus spezifische Wabenkarten bei) oder die weißrussische Arbeit Sprachgeographie und Einteilung der weißrussischen Mundarten (Avanesov, Atrachovič, Mackevič: 1968, 1969). Die Isoglossenbündel in tschechischen Dialekten werden im Tschechischen Sprachatlas (Balhar: 1992-2005), dessen Mitautorin ich bin, vorgestellt, und zwar in seinem 5. Band. Auf 15 Kartenseiten (S. 637-651) werden hier ca. 60 Isoglossenbündel unterschiedlicher Stärke präsentiert. Diese Isoglossenbündel-Karten beruhen auf der Exzerption des ganzen fünfbändigen Atlaswerkes (knapp 1600 Karten). Es wurde festgestellt, dass sich in jedem Bündel Isoglossen mehrerer Sprachebenen schichten: die der phonologischen, morphologischen sowie lexikalischen.

Es ist zu betonen, dass eine Dialektgrenze nicht nur eine bloße „Dialektschei$\mathrm{de}^{\text {“ } z w i s c h e n ~ z w e i / m e h r e r e n ~ S p r a c h e r s c h e i n u n g e n, ~ s o n d e r n ~ a u c h ~ e i n e ~ G r e n z e ~}$ zwischen zweierlei Ausformungen von Sprachbewusstsein ist. Ein Sprachbewusstsein an beiden anliegenden Seiten der Grenze ist dabei weniger stabil, als es im Zentrum (Kern) des Areals der Fall ist. Das äußert sich an den beiden Seiten der Grenzlinie sehr zutreffend, nämlich durch die Existenz von Dubletten (Tripletten). Auf den Isoglossenbündelkarten des Tschechischen Sprachatlasses wird diese Instabilität graphisch durch Schraffur dargestellt (Abb. 1): Mittels dieser Schraffur wird der parallele Verlauf einzelner Isoglossen vorgeführt; die aufgezeichneten vier Isoglossen dienen hier nur als konkrete Beispiele, vgl. auf der Karte unter 450.5 die lexikalische (hi̊lka x čagan 'Stock', kadlátka x trnka 'Pflaume') und phonologische Opposition (die in diesem Fall u. a. als wichtig 
für das gesamtslawische Areal gelten: vosa x osa 'Vespe', mímu (< mému) x mojemu 'Dativform des Pronomens mein').

\section{Isoglossenbündel als Ausgangspunkt zur Dialektanalyse}

In Übereinstimmung mit der Wellentheorie können auf Grund solcher Karten sowohl der Kern und die Peripherie eines Areals als auch Brennpunkte dialektaler Veränderungen festgestellt werden. Gleichfalls kann man Vermutungen über die Herkunft vieler Formen, Formanzien und Ausdrücke anstellen. Als anschauliches Beispiel lassen sich tschechische dialektale Benennungen für Haut auf der gekochten Milch erwähnen (Kloferová 2006): Gerade die exzentrische Stellung der Dialektismen škrábek/škrábka bzw. škrbán/škrban an der Peripherie des Areals mit der Majoritätsbezeichnung škraloup (dies zu usl. *skora 'Rinde, Schale, Haut': Machek 547 s.v. skora) berechtigt uns, eine Vermutung über die gemeinsame Herkunft aller angeführten Wörter auszusprechen - nämlich über ihre Beziehung zum Wort škraloup. Dieses Appellativum kommt heute nämlich einem geläufigen tschechischen Muttersprachler motivisch intransparent vor. Infolgedessen kann man mit der Wirkung der sog. Volksetymologie rechnen. Die angeführten Dialektismen in ihrer heutigen Gestalt sind dann durch Anlehnung an das Verb škrábat 'kratzen' höchstwahrscheinlich entstanden.

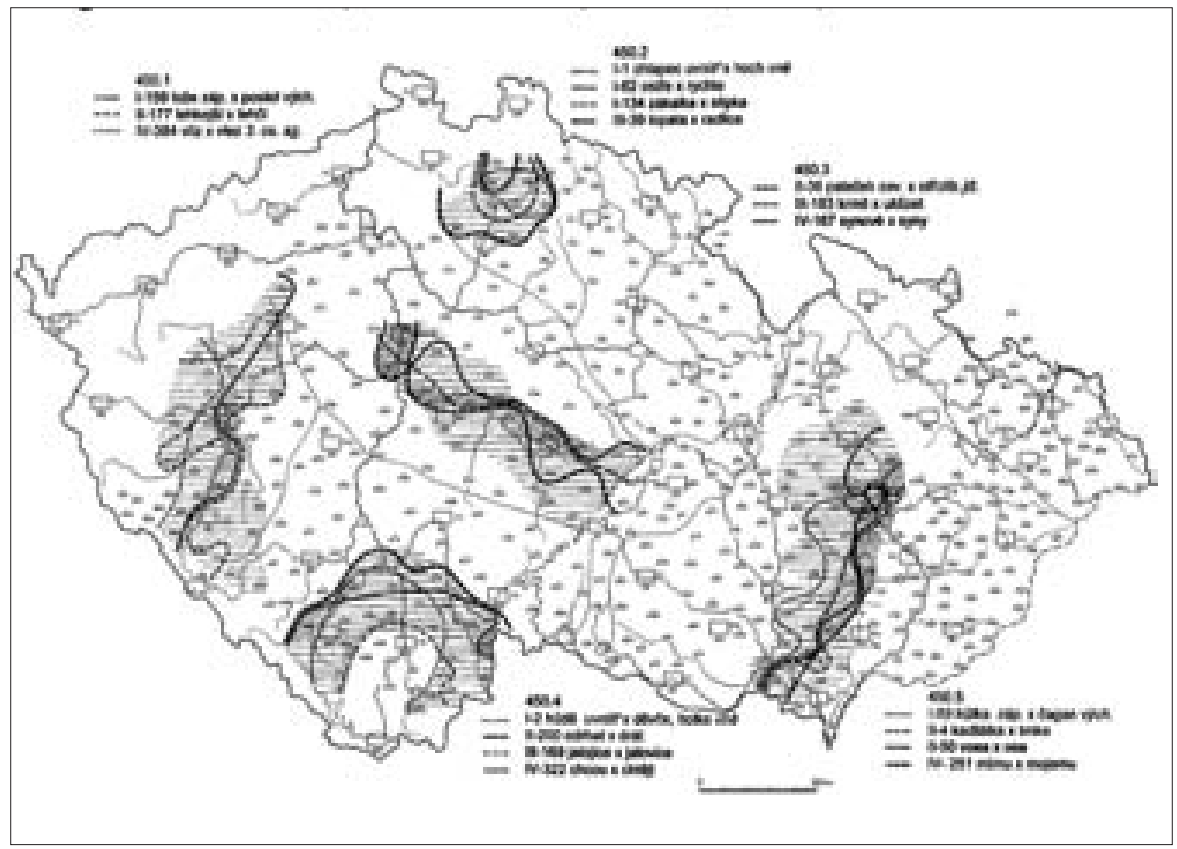

Abb. 1: Isoglossenbündel (Tschechischer Sprachatlas, Bd. 5) 


\section{Propria auf einer Sprachkarte: onymische Areale}

Bisher war die Rede über Areale, die „appellativisch“ im weitesten Sinne des Wortes, also nicht „proprial“" sind: Ihre Grenze wird sowohl durch lexikalische appellativische Isoglossen, $\mathrm{d}$. h. Isolexe, als auch durch Isomorphe sowie Isophone gebildet. Der lexikalische Wortschatz bezieht jedoch zugleich die propriale Sphäre mit ein. Wie bekannt, können auch Propria auf einer Karte dargestellt werden, und zwar auf der onymischen. Natürlich lassen sich auch propriale/onymische Areale abgrenzen. Beide Kartentypen, die proprialen und nichtproprialen, haben sicher ihre Spezifika (bei ihrer kartographischen Zusammenstellung werden unterschiedliche Methoden der Schilderung des Sprachmaterials verwendet) - mit dieser Problematik möchte ich mich jedoch nicht befassen, sie wurde letzten Endes in vielen Studien ausreichend erforscht.

Mit der Beziehung zwischen den proprialen/onymischen und nichtproprialen Arealen im tschechischen Sprachmilieu befasst sich die tschechische Onoma-

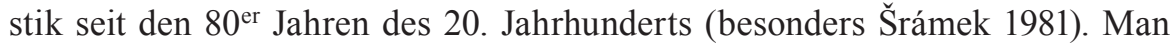
spricht einerseits von einem onymischen, andererseits von einem dialektalen Areal; meiner Meinung nach ist der Terminus „dialektales Areal“ in diesem Zusammenhang nicht allzu passend, weil die Propria einen fixen Bestandteil der Sprache/des Dialekts bilden. Der grundlegende Unterschied zwischen beiden Sphären, der proprialen und der nichtproprialen, besteht im Charakter des Denotats/des zu benennenden Objektes (Einmaligkeit x Allgemeinheit). Dies erweist sich dann in den Methoden der Darstellung auf der Sprachkarte: Eine nichtpropriale Karte kann auf einem Material beruhen, das in einem Netz der ausgewählten Ortschaften gewonnen wurde (ein zu benennendes Denotat/Objekt ist Repräsentant einer Gattung und als solches wird auch seine Bezeichnung als allgemein wahrgenommen/empfunden), während auf einer proprialen/onymischen Karte das gesamte Material geschildert werden muss (ein Proprium, z. B. ein Personenname oder ein Flurname, ist mit einem konkreten onymischen Objekt, d. h. mit einer konkreten Person, mit einem konkreten Feld, mit einer konkreten Wiese etc., in einem konkreten Ort verbunden); vgl. Pleskalová 1981: 167, Harvalík 2004: 37-38.

\section{Appellativisches und onymisches Areal als Ausgangspunkt zur Konfrontation}

Onomastische Studien, die sich diesem Problembereich widmen, richten sich besonders auf die Appellative in ihrer geographischen Verbreitung einerseits und auf die Flurnamen, die diese - von ihrer Herkunft her - Appellative als Bestandteil enthalten, andererseits (in der tschechischen Onomastik v.a. Pleskalová, 1981, Harvalík 2004: 48-62), bzw. wird auch die areale Verbreitung der Wortbildungsformanzien einander gegenübergestellt (so vergleicht z. B. Šrámek, 1999: 192 die appellativischen und toponymischen Suffixe -ištěl-isko; usl. Formans *-isk-). Es zeigt sich, dass die deappellativischen Substantive als 
Bestandteil der Flurnamen bisweilen mittels der Flurnamen in einer anderen territorialen Verbreitung auftreten: In dieser toponymischen Funktion können sie ihre ursprüngliche (appellativische) Verbreitung (sei es die größere oder kleinere) bewahren; vgl. Kloferová 2008: 100-101.

Die Schilderung des toponymischen Materials auf einer onymischen Karte wird auch während Arbeiten am Wörterbuch der Flurnamen in Mähren und Schlesien appliziert (dieses wird von uns, Dialektologen in Brno, vorbereitet). Einer der Vorgänge, den wir benutzen, ist die Möglichkeit, interaktive anoikonymische Karten zusammenzustellen; solche Karten bieten nämlich manchmal Indizien zur Erläuterung eines konkreten Flurnamens an.

\section{Isoglossenbündel als Ausgangspunkt zur Flurnamenanalyse (anhand des Wörterbuchs der Flurnamen in Mähren und Schlesien)}

Im Zusammenhang mit den Isoglossenbündeln kann hier jedoch eine weitere Frage gestellt werden: Ist es möglich, diese Bündel, d.h. die Grenzen, die durch jene Bündel bestimmt werden, auch bei der Flurnamenanalyse zu nutzen? Meiner Meinung nach ja. Eine der Möglichkeiten ist die Erläuterung der etymologisch wenig transparenten Flurnamen.

Z. B. repräsentiert das Bündel Nr. 450.5 (Abb. 1) eine Scheide, die zu den am stärksten ausgeprägten Arealgrenzen auf dem tschechischen Dialektgebiet gehört, wie sie anhand des Atlasses festgestellt wurden. Man kann das Bündel auch als Argument bei der Erläuterung eines Flurnamens verwenden, wie ich nun zeigen möchte:

\section{a) Flurnamen mit der Basis bláz(e)n- (tsch. Appell. blázen 'Narr, Verrück-} te')

In unserem Wörterbuch der Flurnamen in Mähren und Schlesien gibt es z. B. Flurnamen, die sich auf die substantivische Basis blázen (psl. *blazns 'Narr, Verrückter') beziehen: Blázen, Bláznice (primär appellativisch 'Irrenanstalt'), Blázinec ('Irrenanstalt; Rummel, Wirbel'), Bláznivec ('Narr': dies erst zum Adjektivum bláznivý 'narrisch' < blázen). Mittels dieser Flurnamen werden immer Felder benannt. Bisher wurde ihre Motivierung mit den Menschen, die an den schwierig zu bebauenden/kultivierenden Grundstücken gearbeitet haben, in Zusammenhang gestellt: „Die Menschen wurden hier von ihrer Umgebung für Narren gehalten". Vergleicht man jedoch die onymische Karte der angeführten Flurnamen mit dem erwähnten Isoglossenbündel, so stellt man fest, dass die Flurnamen im Bereich dieses Bündels (und zugleich also im Bereich einer starken Arealgrenze) vorkommen (Abb. 2). Nun ist noch eine andere onymische Karte „zu Hilfe zu rufen“: Diese Karte wurde im Buch von R. Šrámek, 1999 publiziert. Es geht um eine Karte der Flurnamen und Ortsnamen, die auf die Basis laz- (psl. *lazъ 'ein durch die Rodung des Waldes entstandenes Feld/Grundstück' (Machek, 1968) zurückzuführen sind (Šrámek 1999: 199; Abb. 3). Man sieht, dass die erwähnten Flurnamen, die in 


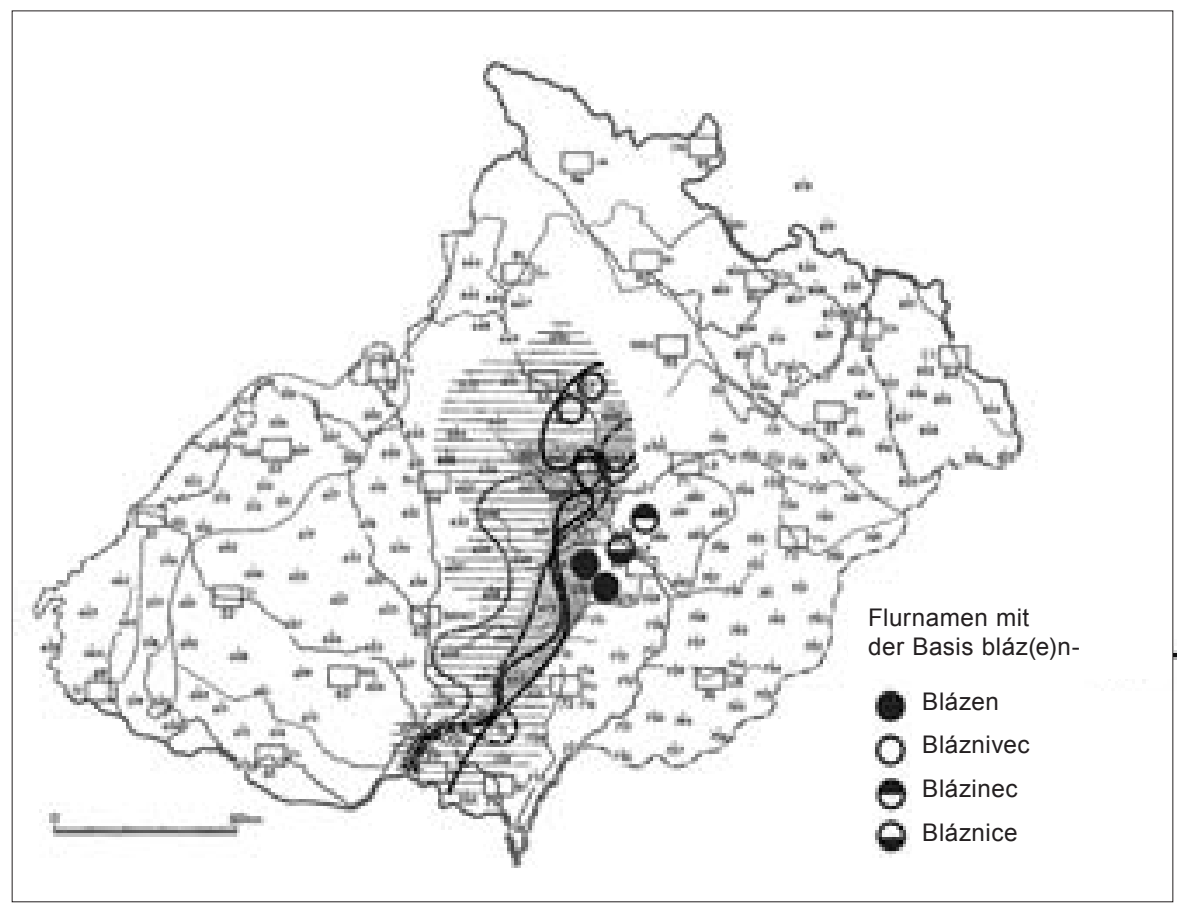

Abb. 2: Isoglossenbündel Nr. 450.5 (Tschechischer Sprachatlas, Bd. 5)

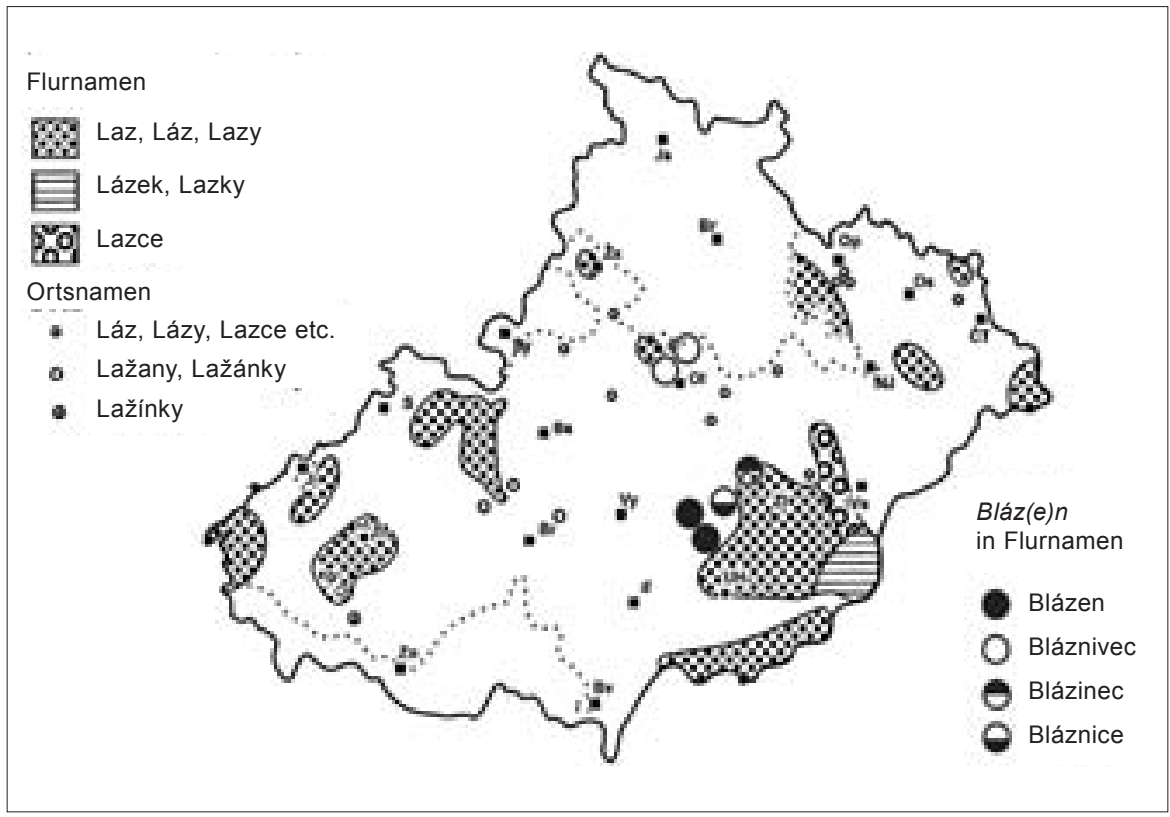

Abb. 3: Laz- (-á-) in Flurnamen und Ortsnamen (Nach Šrámek, 1999) 
unserem Wörterbuch mit der Basis blázen verbunden werden, bloß am Rande der laz-Areale auftreten. Auf Grund dieser Zusammenhänge kann man annehmen, dass den heutigen Flurnamen mit der vermuteten Basis blázen eher eine altertümliche Basis laz- vorangeht. Die Basis laz-gilt jedoch heute für den alltäglichen Sprachgebrauch als unklar, unmotiviert. Gerade diese Isoliertheit hat ermöglicht, die Flurnamen mit laz- unter der Mitwirkung der Volksetymologie zur semantischen Gruppe der Wörter mit bláz- im Anlaut (blázen 'Narr' etc.) zuzuordnen. Diese Erläuterung wird auch durch ein anderes Argument unterstützt: In der Anoikonymie Mährens erscheint das Motiv 'blöd, dumm' überhaupt nicht (z. B. Flurnamen zur Basis hloupý, usl. glupъ, wie *Hlupák, *Hlupaňa etc.).

\section{b) Flurname Balik (tsch. Appell. balik 'Paket')}

Derselbe Vorgang lässt sich höchstwahrscheinlich auch bei der Erläuterung des Flurnamens Balik (für eine Weide) applizieren. Die bisherige Erläuterung rechnet mit der Motivierung 'Paket; etwas Eingepacktes, Verschnürrtes und zum Transport Bestimmtes'. Diesen Flurnamen gibt es am Rand eines Areals, das auch durch ein starkes Isoglossenbündel abgegrenzt ist. Gerade in diesem Areal erscheinen jedoch auch Flurnamen Bál, Bala, Baly (Plt.) und Balinka - in der Regel handelt es sich von den Namen für Felder: Sie sind mit der Basis bal- (vielleicht zum usl. *bala 'hell, glänzend, strahlend, weiß; ein nasser, sumpfiger Ort') zu verbinden. Die angeführte Basis kommt jedoch im gegenwärtigen appellativischen Wortschatz nicht mehr vor. Gerade das onymische Areal der Flurnamen mit Bal- im Anlaut (Bál, Bala etc.) ermöglicht uns zu vermuten, dass auch der Flurnamen Balik (primäre appellativische Bedeutung 'Paket'), der sich am Rand dieses Gebietes befindet, diesem Areal angehört. Unsere neue Erläuterung würde dann keine Übertragung, sondern die Struktur Bal-ik voraussetzen - und dadurch auch die Zugehörigkeit zum semantischen Feld 'hell, glänzend, weiß, nass' etc. Man findet auch kaum ein logisches Motiv (das Tertium comparationis) für die Übertragung des Appellativums balik 'Paket' auf einen Namen für Weide. Die Undurchsichtigkeit/Intransparenz der Basis im gegenwärtigen appellativischen Wortschatz war ein Nährboden für die Volksetymologie, dank welcher der Flurnamen ins Sprachsystem als transparent und motiviert eingegliedert wurde.

\section{Zum Schluss}

Die räumliche Projektion des proprialen und nichtproprialen Materials erlaubt es, Beziehungen zwischen beiden lexikalischen Ebenen, proprialen und nichtproprialen/appellativischen, effektiver zu nutzen. Eine dialektale Grenze, die auf Grund der nichtproprialen Spracherscheinungen geformt wurde, kann für die Erforschung von Erscheinungen proprialen Charakters nützlich sein. Beide Sphären, die propriale sowie die nichtpropriale, sind doch Sphären ei- 
nes Sprachsystems, und als soche können sie sich nicht getrennt voneinander entwickeln. Rechnet man mit ihrer gegenseitigen Gebundenheit, so kann man beide Sphären zu ihrem beiderseitigen Nutzen tiefer kennen lernen.

\section{LITERATUR}

Jan BALHAR et al., 1992-2005: Český jazykový atlas 1-5. Praha: Academia.

Milan HARVALÍK, 2004: Synchronní a diachronni aspekty české onymie. Praha: Academia.

Stanislava KLOFEROVÁ, 2006: Areálová lingvistika: Svazky izoglos ve službách etymologie. Studia etymologica Brunensia 3. Eds. Ilona Janyšková, Helena Karlíková. Praha, Nakladatelství Lidové noviny. 143-148.

Stanislava KLOFEROVÁ, 2008: Česká dialektologie ve slovanském kontextu. Česká slavistika. Př́spěvky k XIV. mezinárodnímu sjezdu slavistů, Ochrid 10.-16. 9. 2008 (= Slavia 77). 93-103.

Václav MACHEK, 1968: Etymologický slovník jazyka českého. Praha: Academia.

Jana PLESKALOVÁ, 1981: K povaze lexikálních diferencí dialektových a onymických. Nazewnictwo obszarów językowo mieszanych. Ed. Hubert Górnowicz. Wrocław - Kraków - Warszawa - Gdańsk: Zakład Narodowy Imienia Ossolińskich. 167-174.

Rudolf ŠRÁMEK, 1981: Areál onymický a areál dialektový. Nazewnictwo obszarów jezzykowo mieszanych. Ed. Hubert Górnowicz. Wrocław - Kraków - Warszawa Gdańsk: Zakład Narodowy Imienia Ossolińskich. 157-166.

Rudolf ŠRÁMEK, 1999: Úvod do onomastiky. Brno: Masarykova univerzita.

Рубен И. АВАНЕСОВ, Кандрат К. АТРАХОВІЧ, Юзефа Ф. МАЦКЕВІЧ [Ruben I., AVANESOV, Kandrat K. ATRACHOVIČ, Juzefa F. MACKEVIČ, J. F.] (eds.), 1968, 1969: Лінгвістычна геаграфія і групоўка беларускіх гаворак [Linhvistyčna heahrafija i hrupoŭka belaruskich havorak] 1, 2. Мінск [Minsk]: Навука і тёхніка [Navuka i technika].

Helmut FASSKE, Helmut JENTSCH, Siegfried MICHALK, 1965-1996: Sorbischer Sprachatlas / Sěrbski rěčny atlas 1-15. Bautzen: Domowina-Verlag.

Jozef ŠTOLC, Ferdinand BUFFA, Anton HABOVŠTIAK, 1968: Atlas slovenského jazyka 1. Vokalizmus a konzonantizmus. Bratislava: Veda.

Anton HABOVŠTIAK, 1984: Atlas slovenského jazyka 4. Lexika. Bratislava: Veda. 


\section{LASTNO- IN OBČNOIMENSKI JEZIKOVNI AREALI: ENAKOST V RAZLIČNOSTI}

Areal označuje celostno lingvistično, etnografsko in zgodovinsko kategorijo, ki jo določamo kot prostor, v katerem se pojavlja/jo določena/e značilnost/i. Področne meje razločimo glede na izoglose ali snope izoglos. Karte v jezikoslovnih atlasih kažejo, da je področna meja ostrejša, če je število skupnih značilnosti večje.

Peti del Češkega lingvističnega atlasa (Czech Linguistic Atlas) predstavlja približno 60 takih snopov. Večinoma gre za področja višjega reda, ki so razmejena s snopi izoglos vseh jezikovnih ravni, središče in obrobje danega področja pa je razmejljivo. To dejstvo je pomembno, kadar primerjamo lastno- in občnoimenske areale - področje določenega toponimskega tipa ima vzporednico/utemeljitev na občnoimenskem področju in obratno. Gre za medsebojno koristen odnos, saj ga lahko uporabimo pri jezikoslovni analizi značilnosti, ki jih je sicer težko pojasniti ali pa so izolirane (npr. prikaz v kartah Atlasa in v kartah, ki so nastale za slovar moravskih in šlezijskih anojkonimov - Dictionary of Moravian and Silesian Anoikonyms). Kot primer lahko navedemo prikaz več čeških anojkonimov (npr. imen polj), ki so izvorno očitno metaforični: Blázen, Bláznivec (primarni apelativ, ki pomeni hkrati ‘blaznež, blazna oseba') in Blázinec, Bláznice (primarni apelativ, ki pomeni hkrati 'norišnico; zmedo'). Anojkonimi so potrjeni na področjih, ki predstavljajo opazne meje med narečji, in na področjih, kjer nastopajo toponimi s korenom laz- (skupni slovanski *lazъ 'polje ali zemlja, nastala s krčenjem gozda'). Koren laz- glede na motivacijo ni več transparenten v sedanjem apelativnem leksikonu. Anojkonimi Blázen, Blázinec itd., ki vsebujejo ta izoliran koren, so nedvomno rezultati zmotne asociacije besed z apelativi s transparentno osnovo bláz(e)n- 'blaznež'. 